\title{
ON SUMS OF POWERS IN RAMANUJAN'S LOST NOTEBOOK
}

\author{
BRUCE C. BERNDT AND DANIEL SCHULTZ
}

\section{Introduction}

On a page published with Ramanujan's lost notebook [2, p. 338], Ramanujan records the seven equalities

$$
\begin{aligned}
& x+(x-1)+(x-2)+\cdots=\frac{1}{2} x^{2}+\frac{1}{2} x+\left\{\begin{array}{l}
+\frac{1}{8}, \\
+0,
\end{array}\right. \\
& x^{2}+(x-1)^{2}+(x-2)^{2}+\cdots=\frac{1}{3} x^{3}+\frac{1}{2} x^{2}+\frac{1}{6} x+\left\{\begin{array}{l}
+\frac{1}{36 \sqrt{3}}, \\
-\frac{1}{36 \sqrt{3}},
\end{array}\right. \\
& x^{3}+(x-1)^{3}+(x-2)^{3}+\cdots=\frac{1}{4} x^{4}+\frac{1}{2} x^{3}+\frac{1}{4} x^{2}+\left\{\begin{array}{l}
+0, \\
-\frac{1}{64},
\end{array}\right. \\
& x^{4}+(x-1)^{4}+(x-2)^{4}+\cdots=\frac{1}{5} x^{5}+\frac{1}{2} x^{4}+\frac{1}{3} x^{3}-\frac{1}{30} x+\left\{\begin{array}{l}
+\frac{1}{900} \sqrt{15+4 \sqrt{\frac{6}{5}}}, \\
-\frac{1}{900} \sqrt{15+4 \sqrt{\frac{6}{5}}},
\end{array}\right. \\
& x^{5}+(x-1)^{5}+(x-2)^{5}+\cdots=\frac{1}{6} x^{6}+\frac{1}{2} x^{5}+\frac{5}{12} x^{4}-\frac{1}{12} x^{2}+\left\{\begin{array}{l}
+\frac{1}{128}, \\
+0,
\end{array}\right. \\
& x^{6}+(x-1)^{6}+(x-2)^{6}+\cdots=\frac{1}{7} x^{7}+\frac{1}{2} x^{6}+\frac{1}{2} x^{5}-\frac{1}{6} x^{3}+\frac{1}{42} x+
\end{aligned}
$$

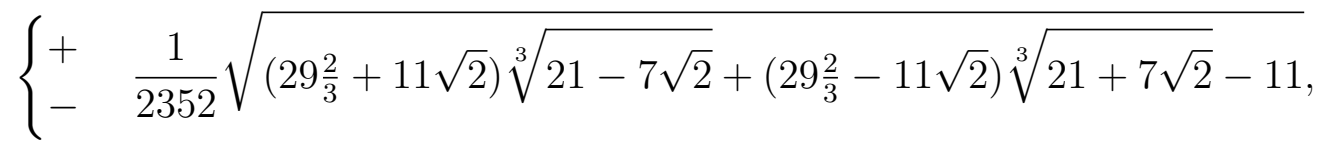

$$
\begin{aligned}
& x^{7}+(x-1)^{7}+(x-2)^{7}+\cdots=\frac{1}{8} x^{8}+\frac{1}{2} x^{7}+\frac{7}{12} x^{6}-\frac{7}{24} x^{4}+\frac{1}{12} x^{2}+\left\{\begin{array}{l}
+0, \\
-\frac{17}{2048} .
\end{array}\right.
\end{aligned}
$$

After these equalities, G. H. Hardy has appended a handwritten note, "I'm not clear what this means."

Because Ramanujan had recorded these equations only for his own use, he did not provide interpretations for the left-hand sides or for the brackets on the right-hand sides. However, there is an interpretation that is consistent with the displayed equations (except for a slight error in the equation for sixth powers). We interpret the left-hand side of each equation as the sum of powers of only positive terms, and we interpret the right-hand side as a polynomial $p_{n}(x)$ plus bounds for the "error term" $R_{n}(x)$, which 
we shall explain later. Thus, write

$$
s_{n}(x):=\sum_{i=0}^{\lfloor x\rfloor}(x-i)^{n}=p_{n}(x)+R_{n}(x) . \quad 1 \leq n \leq 7 .
$$

\section{Verifications of Ramanujan's Claims}

Ramanujan is claiming values for the extrema of $R_{n}(x)$ given by the upper and lower values in the brackets. To confirm this, notice first that if $x$ is a positive integer, then

$$
p_{n}(x)=\sum_{j=0}^{x} j^{n}
$$

Secondly,

$$
\begin{aligned}
s_{n}(x+1) & =\sum_{j=0}^{\lfloor x+1\rfloor}(x+1-j)^{n} \\
& =\sum_{j=0}^{\lfloor x\rfloor+1}(x+1-j)^{n} \\
& =(x+1)^{n}+\sum_{j=1}^{\lfloor x\rfloor+1}(x+1-j)^{n} \\
& =(x+1)^{n}+s_{n}(x) .
\end{aligned}
$$

We also know that the polynomial $p_{n}(x)$ satisfies the same functional equation for all natural numbers, namely, $p_{n}(x+1)=(x+1)^{n}+p_{n}(x)$. However, since a polynomial is uniquely determined by its values on a finite set of points, $p_{n}(x+1)=(x+1)^{n}+p_{n}(x)$ for all $x$. This and (2.2) then imply that $R_{n}(x+1)=R_{n}(x)$. So, we can compute its extrema by examining $R_{n}(x)$ on the interval [0,1]. Observe that for $0 \leq x<1$,

$$
R_{n}(x)=x^{n}-p_{n}(x)
$$

and so the minimum and maximum can be found from elementary calculus.

We now reformulate Ramanujan's assertions in terms of the familiar Bernoulli polynomials $B_{n}(x), n \geq 0$, which are defined by

$$
\frac{t e^{x t}}{e^{t}-1}=\sum_{n=0}^{\infty} B_{n}(x) \frac{t^{n}}{n !}, \quad|t|<2 \pi .
$$

Recall that the Bernoulli numbers $B_{n}, n \geq 0$, are defined by $B_{n}=B_{n}(0), n \geq 0$. It is easy to show that for $n \geq 1, B_{2 n+1}=0$. It is well known that [1, p. 804, Eq. 23.1.4]

$$
\sum_{j=1}^{m} j^{n}=\frac{B_{n+1}(m+1)-B_{n+1}}{n+1}, \quad m, n \geq 1 .
$$

Furthermore [1, p. 804, Eqs. 23.1.5, 23.1.6, 23.1.8]

$$
B_{n}^{\prime}(x)=n B_{n-1}(x), \quad n \geq 1,
$$




$$
B_{n}(x+1)-B_{n}(x)=n x^{n-1}, \quad n \geq 0,
$$

and

$$
B_{n}(1-x)=(-1)^{n} B_{n}(x), \quad n \geq 0 .
$$

In particular, $B_{n}(1)=(-1)^{n} B_{n}, n \geq 0$, which, since $B_{2 n+1}=0$, for $n \geq 1$, implies that

$$
B_{n}(1)=B_{n}, \quad n \geq 2 .
$$

By (2.3), (2.1), (2.4), and (2.6)

$$
\begin{aligned}
R_{n}(x) & =x^{n}-\frac{B_{n+1}(x+1)-B_{n+1}}{n+1} \\
& =x^{n}-\frac{B_{n+1}(x)-(n+1) x^{n}-B_{n+1}}{n+1} \\
& =\frac{B_{n+1}-B_{n+1}(x)}{n+1} .
\end{aligned}
$$

Hence, by (2.5),

$$
R_{n}^{\prime}(x)=-\frac{B_{n+1}^{\prime}(x)}{n+1}=-B_{n}(x), \quad n \geq 1 .
$$

Also, from (2.9) and (2.8),

$$
R_{n}(0)=0, \quad \text { and } \quad R_{n}(1)=0, \quad n \geq 1 .
$$

Lastly, since [1, p. 805, Eq. 23.1.21],

$$
B_{n}\left(\frac{1}{2}\right)=-\left(1-2^{1-n}\right) B_{n}, \quad n \geq 0,
$$

we can conclude from (2.9) that

$$
R_{n}\left(\frac{1}{2}\right)=\frac{2-2^{1-n}}{n+1} B_{n+1} .
$$

We now establish Ramanujan's claims. We first examine the sums for odd powers $n$.

For $n=1$, from (2.10),

$$
R_{1}^{\prime}(x)=-B_{1}(x)=\frac{1}{2}-x,
$$

which has the critical point $x=\frac{1}{2}$. Since $B_{2}=\frac{1}{6}$, we see from $(2.13)$ that $R_{1}\left(\frac{1}{2}\right)=\frac{1}{8}$. Since $R_{1}(0)=R_{1}(1)=0$ by (2.11), Ramanujan's first claim is established.

Let $n=3$. From (2.10),

$$
R_{3}^{\prime}(x)=-B_{3}(x)=-x(x-1)\left(x-\frac{1}{2}\right)
$$

Since the critical points are 0,1 , and $\frac{1}{2}$, since $R_{3}(0)=R_{3}(1)=0$ by $(2.11)$, and since $R_{3}=-\frac{1}{64}$ by $(2.13)$, because $B_{4}=-\frac{1}{30}$, we verify Ramanujan's assertion for third powers.

Put $n=5$. Then from $(2.10)$,

$$
R_{5}^{\prime}(x)=-B_{5}(x)=-x(x-1)\left(x-\frac{1}{2}\right)\left(x^{2}-x-\frac{1}{3}\right),
$$


which has the critical points $0,1, \frac{1}{2}, \frac{1}{2} \pm \frac{1}{2} \sqrt{\frac{7}{3}}$. Since $B_{6}=\frac{1}{42}$, we find from (2.13) that

$$
R_{5}\left(\frac{1}{2}\right)=\frac{1}{128}=.0078125 \text {. }
$$

Furthermore,

$$
R_{5}\left(\frac{1}{2} \pm \frac{1}{2} \sqrt{\frac{7}{3}}\right)=.00308 \ldots
$$

Hence, the maximum and minimum of $R_{5}(x)$ are $\frac{1}{128}$ and 0 , as claimed by Ramanujan.

For $n=7$, by $(2.10)$,

$$
R_{7}^{\prime}(x)=-B_{7}(x)=-x(x-1)\left(x-\frac{1}{2}\right)\left(x^{4}-2 x^{3}+x+\frac{1}{3}\right),
$$

which has the real roots $0,1, \frac{1}{2}$, and the complex roots

$$
\frac{3 \pm \sqrt{3(9 \pm 2 i \sqrt{3})}}{6} .
$$

Since $B_{8}=-\frac{1}{30}$, we find from (2.13) that

$$
R_{7}\left(\frac{1}{2}\right)=-\frac{17}{2^{11}}
$$

and so the last assertion in Ramanujan's list has been verified.

We now turn to the cases for even powers $n$. First, if $n=2$,

$$
R_{2}^{\prime}(x)=-B_{2}(x)=-x^{2}+x-\frac{1}{6},
$$

which has the roots $\frac{1}{2}\left(1 \pm \sqrt{\frac{1}{3}}\right)$. Thus, from (2.9),

$$
R_{2}\left(\frac{1}{2}\left(1 \pm \sqrt{\frac{1}{3}}\right)\right)=-\frac{1}{3} B_{3}\left(\frac{1}{2}\left(1 \pm \sqrt{\frac{1}{3}}\right)\right)= \pm \frac{1}{36 \sqrt{3}} .
$$

Thus, Ramanujan's claim for the sum of squares has been verified.

If $n=4$, then

which has the roots

$$
R_{4}^{\prime}(x)=-B_{4}(x)=-x^{4}+2 x^{3}-x^{2}+\frac{1}{30},
$$

$$
\frac{15 \pm \sqrt{15(15 \pm 2 \sqrt{30})}}{30} .
$$

Now

$$
R_{5}\left(\frac{15 \mp \sqrt{15(15-2 \sqrt{30})}}{30}\right)= \pm \frac{1}{900} \sqrt{15+4 \sqrt{\frac{6}{5}}}= \pm 0.00489164 \ldots
$$

and

$$
R_{5}\left(\frac{15 \pm \sqrt{15(15+2 \sqrt{30})}}{30}\right)= \pm \frac{1}{900} \sqrt{15-4 \sqrt{\frac{6}{5}}}= \pm 0.00362062 \ldots
$$


Thus, again Ramanujan's claim is justified.

The calculations for the case $n=6$ are the most involved, and this is the only case where the results do not agree with Ramanujan's. Let the roots of the polynomial

$$
R_{6}^{\prime}(x)=-B_{6}(x)=-x^{6}+3 x^{5}-\frac{5 x^{4}}{2}+\frac{x^{2}}{2}-\frac{1}{42}
$$

be denoted by $x_{i}, 1 \leq i \leq 6$. Write the roots of this polynomial as $x_{i}=\frac{1}{2}+y_{i}$, where the $y_{i}$ are roots of

$$
y^{6}-\frac{5}{4} y^{4}+\frac{7}{16} y^{2}-\frac{31}{1344}
$$

The roots of this polynomial can be found by Cardano's formula, and they are

$$
12 y_{i}^{2}=5-2 t_{1}^{2} t_{2}-2 t_{1} t_{2}^{2},
$$

where

$$
t_{1}=\sqrt[3]{\frac{11+6 \sqrt{2}}{7}}, t_{2}=\sqrt[3]{\frac{11-6 \sqrt{2}}{7}} .
$$

Observe that to obtain real roots of $(2.15)$, we need to take the real cube roots for $t_{1}$ and $t_{2}$ in (2.16). We find numerically that these real roots are, say, $y_{1}=0.2524593 \ldots$ and $y_{2}=-0.2524593 \ldots$

We would now like to determine the values of $R_{6}\left(\frac{1}{2}+y\right)$ at these two critical points. To do this, observe that $R_{6}\left(\frac{1}{2}+y\right)$ is a polynomial of the seventh degree in $y$, and that if $y$ is a root of (2.14), we can use this equation to reduce the seventh and sixth powers on $y$ to fifth degree expressions. After elementary algebra, we find that

$$
R_{6}\left(\frac{1}{2}+y_{i}\right)=\frac{y_{i}}{4704}\left(93-392 y_{i}^{2}+336 y_{i}^{4}\right) .
$$

Square both sides, expand, then substitute the values given for $y_{i}^{2}$ from (2.15), and finally reduce the exponents on the $t_{i}$ using the values given in (2.16). We thus find that

$$
R_{6}\left(x_{1}\right)=\frac{1}{2352 \sqrt{3}} \sqrt{259-(9-10 \sqrt{2}) t_{1}^{2} t_{2}-(9+10 \sqrt{2}) t_{1} t_{2}^{2}}=.0037236 \ldots,
$$

and $R_{6}\left(x_{2}\right)$ is the negative of this. An equivalent expression, more in line with that given by Ramanujan, is

$$
\pm \frac{1}{2352 \sqrt{21}} \sqrt{1813-(47+39 \sqrt{2}) \sqrt[3]{21-7 \sqrt{2}}-(47-39 \sqrt{2}) \sqrt[3]{21+7 \sqrt{2}}}
$$

which is not equal to the expression given by him. It therefore appears that Ramanujan made an error in his calculations, which in view of the computational difficulties above is not surprising. The authors had the advantage of being able to use Mathematica. 


\section{REFERENCES}

[1] M. Abramowitz and I. A. Stegun, eds., Handbook of Mathematical Functions, Dover, New York, 1965.

[2] S. Ramanujan, The Lost Notebook and Other Unpublished Papers, Narosa, New Delhi, 1988.

Department of Mathematics, University of Illinois, 1409 West Green Street, UrBANA, IL 61801, USA

E-mail address: berndt@illinois.edu

E-mail address: schult25@illinois.edu 